\title{
Archetypal and New Families with Alexander Disease and Novel Mutations in GFAP
}

\author{
Albee Messing ${ }^{1}$, Rong $\mathrm{Li}^{2}$, Sakkubai Naidu ${ }^{3}$, J. Paul Taylor ${ }^{4}$, Lital Silverman ${ }^{2}$, Daniel Flint ${ }^{2}$, \\ Marjo S. van der Knaap ${ }^{5}$, and Michael Brenner \\ ${ }^{1}$ Waisman Center and Department of Comparative Biosciences, University of Wisconsin- \\ Madison, Madison, WI ${ }^{2}$ Department of Neurobiology, University of Alabama-Birmingham, \\ Birmingham, AL ${ }^{3}$ Neurogenetics Unit, Kennedy Krieger Institute, Baltimore, MD ${ }^{4}$ Department of \\ Developmental Neurobiology, St. Jude Children's Research Hospital Memphis, TN ${ }^{5}$ Department \\ of Child Neurology, VU University Medical Centre, Amsterdam, Netherlands
}

\begin{abstract}
Objective-Alexander disease typically results from dominant mutations in GFAP that arise de novo. However, several instances of multigenerational familial cases have been reported, with some of the most extensive having been described prior to the identification of GFAP as the related gene. These historically seminal families continue to be of interest due to their clinical variability and suggestions of recessive inheritance or germline mosaicism. We here describe genetic analyses of the two most thoroughly studied historical families, as well as one newly discovered family.
\end{abstract}

Design-Clinical histories were obtained and DNA was analyzed from blood, cheek epithelial cells, or fixed paraffin embedded surgical samples.

Subjects-Affected and unaffected adult members of three families and affected children were included.

Results-Family A contains four siblings in whom we found a novel p.Ser247Pro mutation that was paternally inherited. The phenotypes of these siblings include one unaffected adult, one juvenile onset, and two adult onset. Family B spans four generations, including the first described adult-onset patient originally reported in 1968. Analysis of members of the later generations revealed a novel p.Asp417Ala mutation. Family $\mathrm{C}$ contains three generations. We detected a novel p.Gln426Leu mutation that is the farthest C-terminal mutation known.

Conclusions-These families display clear evidence of variable phenotypes, but do not support recessive inheritance. While germline mosaicism cannot be excluded for one family (A), we propose that for genetic counseling purposes the risk of germline mosaicism should be described as less than $1 \%$.

\section{Keywords}

Alexander disease; GFAP; inheritance patterns; expressivity; phenotypic variation 


\section{INTRODUCTION}

Alexander disease is a rare and generally fatal neurodegenerative disorder of the central nervous system that results from mutations in the astrocyte intermediate filament protein, glial fibrillary acidic protein (GFAP). Before the genetic basis of the disease was discovered, diagnosis required autopsy or biopsy identification of Rosenthal fibers, the characteristic cytoplasmic protein aggregates that are found in abundance in subpial, subependymal, and perivascular astrocytes ${ }^{1}$. The most widely recognized form affects young children whose initial signs occur before the age of 2 years and who have short life spans. In 1968, based on the unique pathology of abundant and disseminated Rosenthal fibers, Seil et al. ${ }^{2}$ described the first adult-onset form of the disease. Subsequently other patients with intermediate ages of onset were identified. In 1992, Duckett et al. ${ }^{3}$ described an unusual family with two affected children, one juvenile onset and the other adult onset, both of whom received biopsy confirmation of their diagnoses.

GFAP was discovered as the gene mutated in Alexander disease by a candidate gene approach ${ }^{4}$. Later studies quickly linked early and later onset forms of the disease as having a common origin in mutations in this single gene ${ }^{5}$ (for review, see ${ }^{6}$ ). All known mutations are heterozygous, acting in an autosomal dominant fashion. Nearly all are missense mutations, predicting a change in a single amino acid, although recently several other types of mutations have been described with short in-frame insertions or deletions or frameshifts occurring at the tail end of the protein. Most mutations arise de novo, though in some instances patients live to reproductive age and transmit the mutation to subsequent generations. Germline mosaisicm, which might be the explanation for the family described by Duckett et al. ${ }^{3}$, has yet to be documented for any families where Alexander disease occurred in multiple siblings. The penetrance of Alexander disease approaches $100 \%$, the few exceptions being patients who may have only been pre-symptomatic at the time of study.

Despite the rapid advances of the past decade, the genetic basis for many of the original Alexander disease patients described prior to 2001 has not been established. We now report that GFAP mutations, albeit novel ones, account for the patients described by Seil et al. ${ }^{2}$ and Duckett et al. ${ }^{3}$, as well as previously unreported members of their respective families. Analyses of these families also expands the spectrum of phenotypic variability associated with GFAP mutations, even within families, and indicate that some patients not only stabilize but sustain long periods of clinical improvement. We also present a third family, again with a novel mutation, that prompts consideration of additional phenotypes in future studies of Alexander disease.

\section{MATERIALS AND METHODS}

Informed consent for genetic studies was obtained following protocols approved by the IRB's at the University of Wisconsin-Madison and University of Alabama-Birmingham. DNA was isolated from samples of peripheral blood using the PureGene kit according to the manufacturer's recommendations (Gentra Systems). DNA was isolated from fixed tissues in paraffin blocks as previously described ${ }^{5}$. DNA sequencing was performed as previously described $^{4}$, and the presence of the mutations confirmed by restriction enzyme digestion.

Functional analysis of mutant glial fibrillary acidic proteins: Plasmids used were pcDNA3.1 vectors expressing either the human wild type GFAP coding region (pcDNA3.1-hGF(WT)) or GFAP with the p.Ser247Pro mutation (pcDNA3.1-hGF(S247P)), p.Asp417Ala mutation (pcDNA3.1-hGF(D417A)) or p.Gln426Leu mutation (pcDNA3.1-hGF(Q426L)). Plasmids 
were constructed and GFAP expression analyzed by transfection into SW13 $\mathrm{vim}^{-}$cells as previously described ${ }^{5}$.

Germline inheritance: The parental origin of the chromosome bearing the p.Ser247Pro mutation was determined as previously described, using IVS4+826G $>A$ as the informative $\mathrm{SNP}^{7}$.

\section{RESULTS}

\section{Family A}

Family A consists of eight siblings born to non-consanguineous parents. Two of these siblings were previously reported by Duckett et al. ${ }^{3}$ to document the existence of "familial" Alexander disease. A pedigree for this family is shown in Figure 1A, with birth order disguised to protect confidentiality. Patient II.c presented at 26 years of age with a 1 year history of headaches and incoordination. Her condition progressed and she died at the age of 50. Samples of brain were donated to the Brain and Tissue Bank for Neurodevelopmental Disorders at the University of Maryland (accession \#4858). Patient II.d presented at 8 years of age for chronic vomiting and poor weight gain. At the age of 29.5 years he remains cognitively intact, although he has obstructive sleep apnea, weakness of palatal elevation and diminished gag reflex on the right, causing slight difficulty swallowing liquids. His head circumference is $59.5 \mathrm{~cm}(98 \%)$. Patient II.f presented at the age of 29 years, nine months after experiencing an episode of atonia, with inability to move her arms and legs. She then developed episodic headaches and neck pain, with progressive deterioration in balance. In retrospect she recalled gait abnormalities and dizziness that began during her early 20 's. She had intermittent swallowing difficulties. Her head circumference was $58 \mathrm{~cm}(98 \%)$. MRIs of patient II.c revealed severe atrophy of the medulla oblongata, a lesion in the pons, and abnormal signal in the cerebellar white matter (Figure 2A, $46 \mathrm{yr}$ ), and of patient II.d an enhancing lesion in the medulla oblongata (Figure 2B, $15 \mathrm{yr}$ ). Asymptomatic siblings available for study included II.b and II.e. II.b has no known neurological or cognitive deficits, and is healthy at the age of 51 years. II.e had no known neurological or cognitive deficits, and was fully employed after receiving a Ph.D. in engineering. He died at the age of 43 from suicide.

The parents of these siblings are designated I.b (alive and healthy in her late 60's) and I.a (died at the age of 66 from a myocardial infarction). The father (I.a) had a history of multiple chronic health problems, which included alcoholism and scoliosis of undefined origin that began as a young adult. His children also reported that he had a chronic history of snoring and sleep apnea.

DNA isolated from blood samples was analyzed for GFAP coding mutations for each of the affected children (II.c, II.d, II.f), and two apparently unaffected children (II.b and II.e). For II.d, all nine exons and some flanking intronic segments were sequenced as previously described ${ }^{4}$. A heterozygous $739 \mathrm{~T} \rightarrow \mathrm{C}$ mutation was found in exon 4 which predicts an p.Ser247Pro coding change. Presence of the mutation results in gain of a HaeII restriction site, and this alteration was used to confirm the presence of the mutation. The other family members were similarly tested by restriction enzyme digestion, and the two other affected children (II.c, II.f) also tested positive for the p.Ser247Pro mutation. Interestingly, while one unaffected sib (II.b) was wild-type, the other (II.e) was positive. The presence of the heterozygous mutation in II.c and II.f was confirmed by sequencing.

The finding of multiple siblings in the same family with the same mutation suggests either inheritance from one of the parents or germline mosaicism. To attempt to address this question we initially determined the genotype of the mother, since the father was deceased. 
However, the mother (I.b) was wild-type for both alleles. We then determined which parental allele harbored the mutated GFAP for patient II.d. Eleven of 13 clones tested were consistent with the mutation being on the paternally inherited chromosome, the most frequent pattern for sporadic Alexander disease ${ }^{7}$. We subsequently located paraffin blocks from a testicular biopsy done on the father (I.a) several years before his death, and isolated DNA from this tissue for sequencing. I.a was heterozygous for the p.Ser247Pro mutation.

Family B

Family B consists of four generations, the earlier members of which were originally described by Seil et al. ${ }^{2}$ and Schwankaus et al. ${ }^{8}$. A pedigree for this family is shown in Figure 1B, continuing the nomenclature used by Schwankhaus et al. ${ }^{8}$ to facilitate identification of individuals across publications. The first reported example of adult-onset Alexander disease was patient III.F ${ }^{2}$, who presented at the age of 32 years for rapid onset paralysis of his left arm. He died at the age of 47 and received an autopsy diagnosis of Alexander disease. Two of his brothers, patients III.A and III.E, were considered potentially affected. Three daughters of III.F (IV.B, IV.D, and IV.F) were subsequently described by Schwankaus et al. ${ }^{8}$, one of whom received an autopsy diagnosis (IV.B). Our subsequent studies have concentrated on patient IV.B and her descendents.

Patient V.B had onset at the age of 37 years with generalized weakness that worsened over the course of a year, during which time she also had frequent headaches and experienced significant weight gain (40-50 lb.). She gradually became non-ambulatory and died at the age of 42. Autopsy confirmed the diagnosis of Alexander disease. Samples of brain were donated to the Brain and Tissue Bank for Neurodevelopmental Disorders at the University of Maryland (accession \#5109). Patient VI.A is the nephew of V.B, and presented at the age of 7 years for intractable vomiting. His parents were reported as healthy in their mid-30's. MRIs revealed a lesion in dorsal medulla and pons, with mass effect that caused initial consideration of glioma (Figure 3). Subsequent review revealed signal abnormalities and slight swelling of the caudate nucleus and putamen (Figure 3). The brainstem lesion enhanced after contrast (Figure 3). A second MRI obtained shortly thereafter revealed small frontal white matter lesions, and enhancement of the subependymal region (data not shown). Re-review of an earlier CT taken at the age of 6.5 showed a thin rim of increased density around the lateral ventricles, particularly in the frontal region. The radiological findings were considered diagnostic for Alexander disease. At the age of 10 years a G-tube was inserted, leading to rapid weight gain. At the age of 11 he was reported as asymptomatic, with above-average intelligence.

DNA isolated from blood samples was analyzed for GFAP coding mutations for selected members of generations V and VI in Figure 1B. For patient VI.A, all nine exons and flanking intronic segments were sequenced as previously described ${ }^{4}$. A heterozygous $1250 \mathrm{~A} \rightarrow \mathrm{C}$ mutation in exon 8 was found, which predicts a p.Asp417Ala coding change. Other individuals were tested by BstF51 digestion for the p.Asp417Ala mutation. The clinically affected aunt (V.B) and unaffected father (V.D) of patient VI.A had the same mutation, whereas his mother (V.C) was negative. We subsequently obtained autopsy samples of brain from patient IV.B (the mother of both V.B and V.D, and reported by ${ }^{8}$ ) in the form of paraffin blocks, and isolated DNA from this tissue. This individual was also heterozygous for the p.Asp417Ala mutation. The presence of the mutations in IV.B, V.B, and V.D were confirmed by sequencing.

\section{Family C}

Family $\mathrm{C}$ consists of three generations, two with affected individuals (pedigree shown in Figure 1C). Patient II.1 developed a progressive gait disorder at the age of 46 years. She is 
one of eight siblings, and the only one affected. Initial MRI findings were inconclusive. She suffered significant deterioration after a session of hydrotherapy, and is now wheelchair bound at the age of 50. Patient III.1, one of three daughters of patient II.1, had scoliosis from the age of 9, but no apparent neurological symptoms. She had a history of alcohol abuse and bipolar affective disorder. She died at the age of 21 years, shortly after being found unresponsive with a blood alcohol level of $0.22 \%$. Autopsy revealed characteristic features of Alexander disease, including subpial, subependymal, and perivascular accumulations of Rosenthal fibers.

Initial analysis of GFAP was conducted on a blood sample from patient II.1, prompted by the autopsy findings of Rosenthal fibers in her daughter, and revealed a heterozygous $1277 \mathrm{~A} \rightarrow \mathrm{T}$ mutation in exon 9 predicting a p.Gln426Leu coding change. Samples of spleen embedded in paraffin were subsequently obtained from the autopsy of patient III.1. DNA extraction and genotyping revealed the same p.Gln426Leu mutation as found in her mother. We then obtained DNA samples from the parents of patient II.1 (blood from the mother, I.2, and DNA extracted from paraffin blocks of a surgical sample of prostate from the father, I. 1). Both samples from generation I were negative for the p.Gln426Leu mutation, supporting the conclusion that the mutation in patient II. 1 arose de novo and is thus virtually certain to be disease-causing.

\section{Functional Analysis of the Mutant GFAP Proteins}

Given the variability in phenotypes associated with the p.Ser247Pro and p.Asp417Ala mutations, and the unusual site of the p.Gln426Leu mutation near the C-terminal tail, we tested the effects of these mutations on filament assembly using transfection assays in cultured cells. For these experiments, SW13vim ${ }^{-}$cells, which have no endogenous cytoplasmic intermediate filaments that could complicate the interpretation ${ }^{9}$, were transfected with vectors which expressed either the mutated or wild type human GFAP protein. Transfection with the wild type vector often gave rise to normal GFAP filaments (Figure 4A), but normal filaments were never seen in cells transfected with the p.Ser247Pro mutant; instead, the latter often showed ring-like filaments on a background of diffuse staining (Figure 4B). Since the affected family members are all heterozygous for the p.Ser247Pro change, the effect of cotransfection of the mutant and wild type vectors was also tested. Presence of the wild type protein failed to rescue the defect in GFAP polymerization, consistent with a dominant effect of the p.Ser247Pro mutation (Figure 4B, insert). Similar results were obtained for the p.Asp417Ala and p.Gln426Leu mutant proteins, with the additional feature that an aggregate which appeared to be an agglomeration of rings was often seen extending outwards from the nucleus (Figures 4C-D).

\section{DISCUSSION}

We report genetic findings in three families with Alexander disease, two of which were previously known from seminal clinicopathologic publications during the pre-genetic era. A novel mutation was found for each of the three families. Although the clinical presentations now fit within the recognized spectrum of juvenile and adult-onset Alexander disease ${ }^{10-12}$, consideration of these families raises a number of important issues, such as the criteria for judging causality when confronted by novel mutations, the possibilities of recessive inheritance, and the sometimes extreme variability in expressivity of the clinical phenotypes.

The GFAP mutation in family $\mathrm{C}$ was found to arise de novo in patient II.1, providing strong evidence that it is disease causing. For families A and B the origin of the mutations could not be determined, requiring other evidence to establish their disease causality. Three critical criteria for being disease causing (reviewed in ${ }^{6}$ were met by these mutations: 1 ) they were present in each affected family member tested;2) the DNA changes are not simply single 
nucleotide polymorphisms (SNP), since they have not been reported for over 100 other individuals whose GFAP coding region has now been sequenced for Alexander disease diagnosis, nor are they reported in the NCBI SNP data base, which includes sequence results from 200 Danish controls ${ }^{13}$; 3) each mutant GFAP displays grossly aberrant polymerization when expressed in SW13vim ${ }^{-}$cells.

Each of the new GFAP mutations reported here, Ser $\rightarrow$ Pro, Asp $\rightarrow$ Ala and Gln $\rightarrow$ Leu, involves a significant change in amino acid properties; however, GFAP mutations as conservative as Asp $\rightarrow \mathrm{Glu}$ and $\mathrm{Leu} \rightarrow \mathrm{Val}$ also cause Alexander disease (reviewed in 6). Clearly, the context in which an amino acid substitution occurs is critical. Like other cytosolic intermediate filament proteins, the GFAP monomer has been described as having unstructured $\mathrm{N}$ - and $\mathrm{C}$-terminal domains separated by a central rod region consisting of four a-helical segments, 1A, 1B, 2A and 2B, separated by non-helical linkers L1, L12 and L2. By this scheme the p.Ser247Pro coding change found in family A occurs at the penultimate amino acid in the $2 \mathrm{~A}$ helical segment, and would be expected to shorten the a-helical segment by just two amino acids. However, recent crystallographic evidence suggests a continuity of $\mathrm{a}$-helix from $2 \mathrm{~A}$ to $2 \mathrm{~B}^{14}$, providing a more understandable basis for the p.Ser247Pro change having significant consequences. The p.Asp417Ala and p.Gln426Leu mutations occur in the non-helical tail region, and are the most C-terminal point mutations reported. Asp417 is part of the RDG sequence that is highly conserved among intermediate filaments. Cell transfection experiments have indicated a role for this sequence in GFAP filament formation ${ }^{15}$, and it has also been suggested to participate in interactions with other proteins ${ }^{16}$. The adjacent Arg416 site is a hot spot for Alexander disease mutations ${ }^{6}$ (see also www.waisman.wisc.edu/alexander and www.interfil.org). The p.Gln426Leu mutation in family $\mathrm{C}$ occurs just seven amino acids from the end of the protein, making it the most $\mathrm{C}$ terminal mutation yet described for Alexander disease. No evidence for functionality for this region of the protein has previously been reported; in fact, the entire C-terminal region after the RDG sequence can be deleted from GFAP without affecting its polymerization in transfected SW13 $\mathrm{vim}^{-}$cells ${ }^{15}$. While the drastic effect of a point mutation within this region might be considered surprising (Figure 4D), it is consistent with a previous study of desmin, also a type III intermediate filament, which found effects on assembly from two myofibrillar myopathy-associated point mutations lying C-terminal of the RDG sequence ${ }^{17}$. However, the effects of these two C-terminal desmin mutations were modest, and neither has been shown to arise de novo; thus the de novo occurrence of p.Gln426Leu strengthens the disease-association for coding changes that are $\mathrm{C}$-terminal to the RDG sequence.

Duckett et al. ${ }^{3}$ were one of the first to raise the possibility of recessive inheritance for Alexander disease. They reported a family with two affected siblings, one juvenile and the other adult-onset, both of whom received biopsy confirmation of their diagnoses. However, the past decade has shown that all known disease causing mutations in GFAP are heterozygous. Another possibility to explain multiple siblings with identical mutations is germline mosaicism. While a theoretical possibility, germline mosaicism has not been demonstrated for any family with Alexander disease. Namekawa ${ }^{18}$ reported two brothers with onsets in their 30's and 40's who each had the same p.Arg276Leu mutation. While their parents were described as neurologically normal into their 70's, none of their tissues were available for analysis, leaving the question of germline mosaicism unresolved. Our finding that all affected members of family A harbored heterozygous p.Ser247Pro mutations, also present in testicular tissue from their father, shows that inheritance was dominant rather than recessive. Whether germline mosaicism occurred in this family cannot be determined; the father showed clinical signs consistent with mild impairment, but the only tissue available for analysis was testicular, in which the vast majority of DNA is derived from sperm. Estimating risk of germline mosaicism is particularly problematic for rare disorders, as discussed by Edwards ${ }^{19}$, and it is likely that a parent with mosaicism of 
germ cells would possess some degree of somatic mosaicism as well. Given that over 100 patients/families have now been published who have genetic diagnoses of Alexander disease, with no documented cases of germline mosaicism, we propose that for genetic counseling purposes the risk of germline mosaicism should be described as less than $1 \%$.

While the GFAP mutations causing Alexander disease are typically viewed as $100 \%$ penetrant, several examples now exist that warrant reconsideration of this issue. Previous discussions of variable expressivity have been complicated by uncertainty whether genetically positive individuals were being seen at pre-symptomatic stages, and especially by variability in depth of evaluation. One example is a family with p.Leu331Pro mutations that consists of a 16 month old boy with megalencephaly and MRI changes characteristic of Alexander disease, but who is otherwise asymptomatic ${ }^{20}$. His sister and mother both had the same mutation, and mild changes on MRI, but no megalencephaly or any other symptoms. Whether this particular mutation is unusually mild, or any of these three individuals are truly unaffected, is unclear. A second example is the p.Asp78Glu family reported by Stumpf et al. ${ }^{21}$. Most of these patients were adult-onset, but one individual experienced juvenile onset. In addition, one 30 year old female was identified who was positive for the mutation but without any obvious symptoms. However, she was unavailable for a complete neurological exam. The findings in our p.Asp417Ala family clearly support variable expressivity, at least with respect to combined juvenile and adult onsets in the same family. Our findings in the p.Ser247Pro family also provide support for variable expressivity, not only with regard to having both juvenile and adult onsets among siblings, but a father who was possibly affected and lived until 62 with only mild and equivocal signs of disease. Furthermore, this family included one mutation positive brother who had no neurological symptoms at all until his death at age 43. Unfortunately, this latter individual's tissues were not available for postmortem review, and no ante-mortem imaging had been conducted.

Finally, whether GFAP mutations account for the occasional individuals in whom abundant Rosenthal fibers are found at autopsy without preexisting suspicion of Alexander disease, previously termed "Rosenthal fiber encephalopathy" 22 , is an interesting question. These patients typically had histories of systemic illnesses (such as infection, neoplasia, or hypertension), sometimes combined with substance abuse ${ }^{23}$. An alternative possibility is that secondary illness or intoxications can independently lead to the formation of Rosenthal fibers, perhaps by simple up-regulation of GFAP expression above a critical threshold (as happens in transgenic mice that over-express wild type GFAP ${ }^{24}$ ). These questions should be considered in future research on Alexander disease and the interpretive value of Rosenthal fibers.

\section{Acknowledgments}

This work was supported by grants from the NIH (NS42803, NS22475, HD03352, NS47466, and HD38985). We are extremely grateful to all the patients, family members, and clinicians who have assisted with this study.

\section{REFERENCES}

1. Alexander WS. Progressive fibrinoid degeneration of fibrillary astrocytes associated with mental retardation in a hydrocephalic infant. Brain. 1949; 72:373-381. [PubMed: 15409268]

2. Seil FJ, Schochet SS Jr, Earle KM. Alexander's disease in an adult. Report of a case. Archives of Neurology. 1968; 19:494-502. [PubMed: 5684299]

3. Duckett S, Schwartzman RJ, Osterholm J, Rorke LB, Friedman D, McLellan TL. Biopsy diagnosis of familial Alexander's disease. Pediatric Neurosurgery. 1992; 18:134-138. [PubMed: 1457372]

4. Brenner M, Johnson AB, Boespflug-Tanguy O, Rodriguez D, Goldman JE, Messing A. Mutations in $G F A P$, encoding glial fibrillary acidic protein, are associated with Alexander disease. Nature Genetics. 2001; 27:117-120. [PubMed: 11138011] 
5. Li R, Johnson AB, Salomons G, et al. Glial fibrillary acidic protein mutations in infantile, juvenile, and adult forms of Alexander disease. Annals of Neurology. 2005; 57:310-326. [PubMed: 15732097]

6. Brenner, M.; Goldman, JE.; Quinlan, RA.; Messing, A. Alexander disease: a genetic disorder of astrocytes. In: Parpura, V.; Haydon, PG., editors. Astrocytes in (Patho)Physiology of the Nervous System. New York: Springer; 2009. p. 591-648.

7. Li R, Johnson AB, Salomons GS, et al. Propensity for paternal inheritance of de novo mutations in Alexander disease. Human Genetics. 2006; 119:137-144. [PubMed: 16365765]

8. Schwankhaus JD, Parisi JE, Gulledge WR, Chin L, Currier RD. Hereditary adult-onset Alexander's disease with palatal myoclonus, spastic paraparesis, and cerebellar ataxia. Neurology. 1995; 45:2266-2271. [PubMed: 8848205]

9. Hedberg KK, Chen LB. Absence of intermediate filaments in a human adrenal cortex carcinomaderived cell line. Experimental Cell Research. 1986; 163:509-517. [PubMed: 3956586]

10. Pareyson D, Fancellu R, Mariotti C, et al. Adult-onset Alexander disease: a series of eleven unrelated cases with review of the literature. Brain. 2008; 131:2321-2331. [PubMed: 18684770]

11. Balbi P, Salvini S, Fundarò C, et al. The clinical spectrum of late-onset Alexander disease: a systematic literature review. Journal of Neurology. 2010; 257:1955-1962. [PubMed: 20721574]

12. Prust M, Wang J, Morizono H, et al. GFAP mutations, age of onset, and clinical sub-types in Alexander disease. Annals of Neurology. (in press).

13. Li Y, Vinckenbosch N, Tian G, et al. Resequencing of 200 human exomes identifies an excess of low-frequency non-synonymous coding variants. Nature Genetics. 2010; 42:969-972. [PubMed: 20890277]

14. Nicolet S, Herrmann H, Aebi U, Strelkov SV. Atomic structure of vimentin coil 2. J Struct Biol. 2010 May.170:369-376. [PubMed: 20176112]

15. Chen WJ, Liem RKH. The endless story of the glial fibrillary acidic protein. Journal of Cell Science. 1994; 107:2299-2311. [PubMed: 7983188]

16. Quinlan R. Cytoskeletal catastrophe causes brain degeneration. Nature Genetics. 2001; 27:10-11. [PubMed: 11137988]

17. Bär H, Goudeau B, et al. Conspicuous involvement of desmin tail mutations in diverse cardiac and skeletal myopathies. Human Mutation. 2007; 28:374-386. [PubMed: 17221859]

18. Namekawa M, Takiyama Y, Aoki Y, et al. Identification of GFAP gene mutation in hereditary adult-onset Alexander's disease. Annals of Neurology. 2002; 52:779-785. [PubMed: 12447932]

19. Edwards JH. Familiarity, recessivity and germline mosaicism. Annals of Human Genetics. 1989 Jan.53:33-47. [PubMed: 2658737]

20. Shiihara T, Sawaishi Y, Adachi M, Kato M, Hayasaka K. Asymptomatic hereditary Alexander's disease caused by a novel mutation in GFAP. Journal of the Neurological Sciences. 2004; 225:125-127. [PubMed: 15465095]

21. Stumpf E, Masson H, Duquette A, et al. Adult Alexander disease with autosomal dominant transmission: a distinct entity caused by mutation in the glial fibrillary acid protein gene. Archives of Neurology. 2003; 60:1307-1312. [PubMed: 12975300]

22. Jacob J, Robertson NJ, Hilton DA. The clinicopathological spectrum of Rosenthal fibre encephalopathy and Alexander's disease: a case report and review of the literature. Journal of Neurology, Neurosurgery, and Psychiatry. 2003; 74:807-810.

23. Wilson SP, Alsarraj S, Bridges LR. Rosenthal fiber encephalopathy presenting with demyelination and Rosenthal fibers in a solvent abuser - adult Alexander's disease. Clinical Neuropathology. 1996; 15:13-16. [PubMed: 8998849]

24. Messing A, Head MW, Galles K, Galbreath EJ, Goldman JE, Brenner M. Fatal encephalopathy with astrocyte inclusions in GFAP transgenic mice. American Journal of Pathology. 1998; 152:391-398. [PubMed: 9466565] 
A

1

II

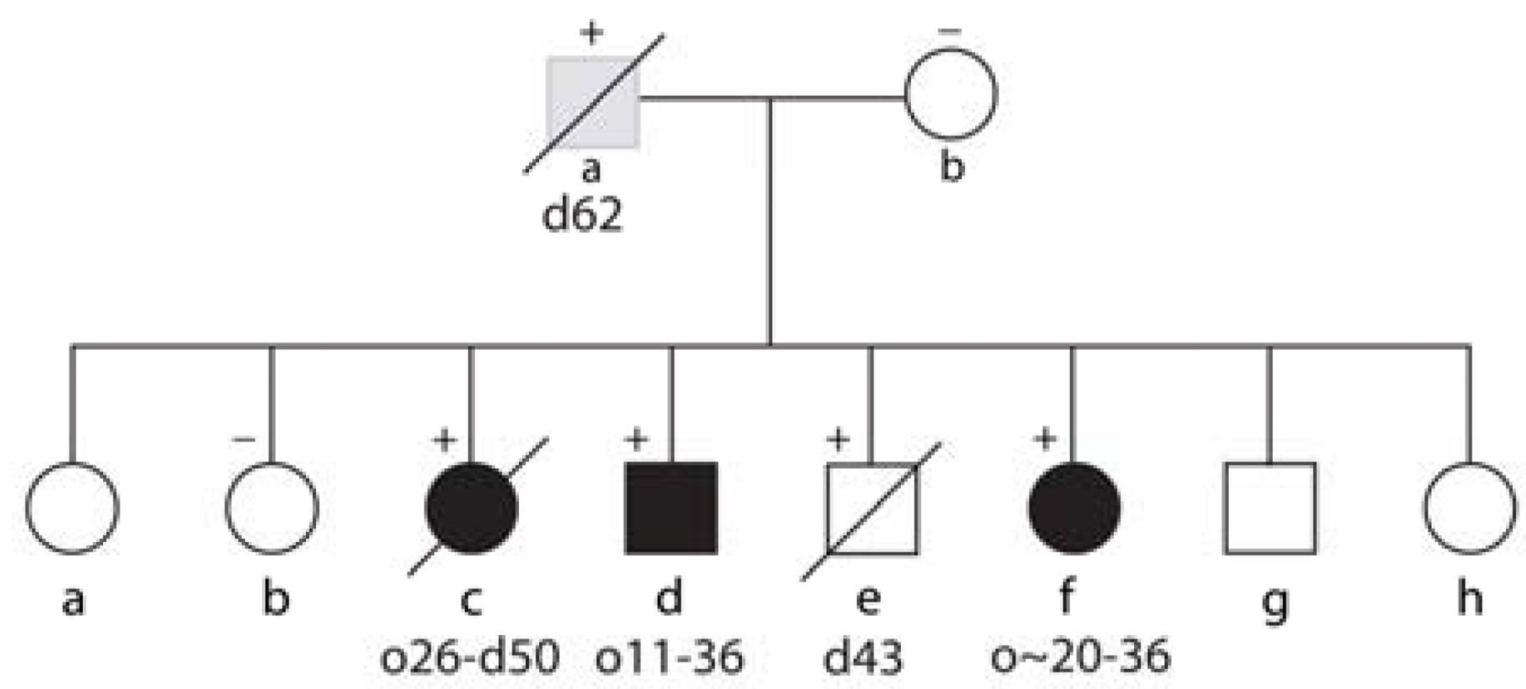


B

III

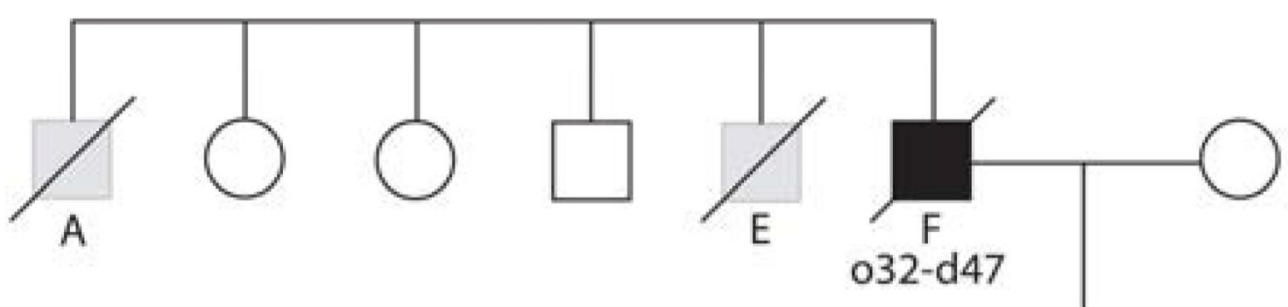

IV
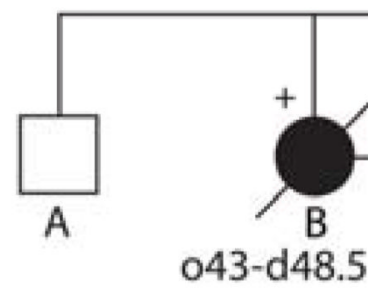

V
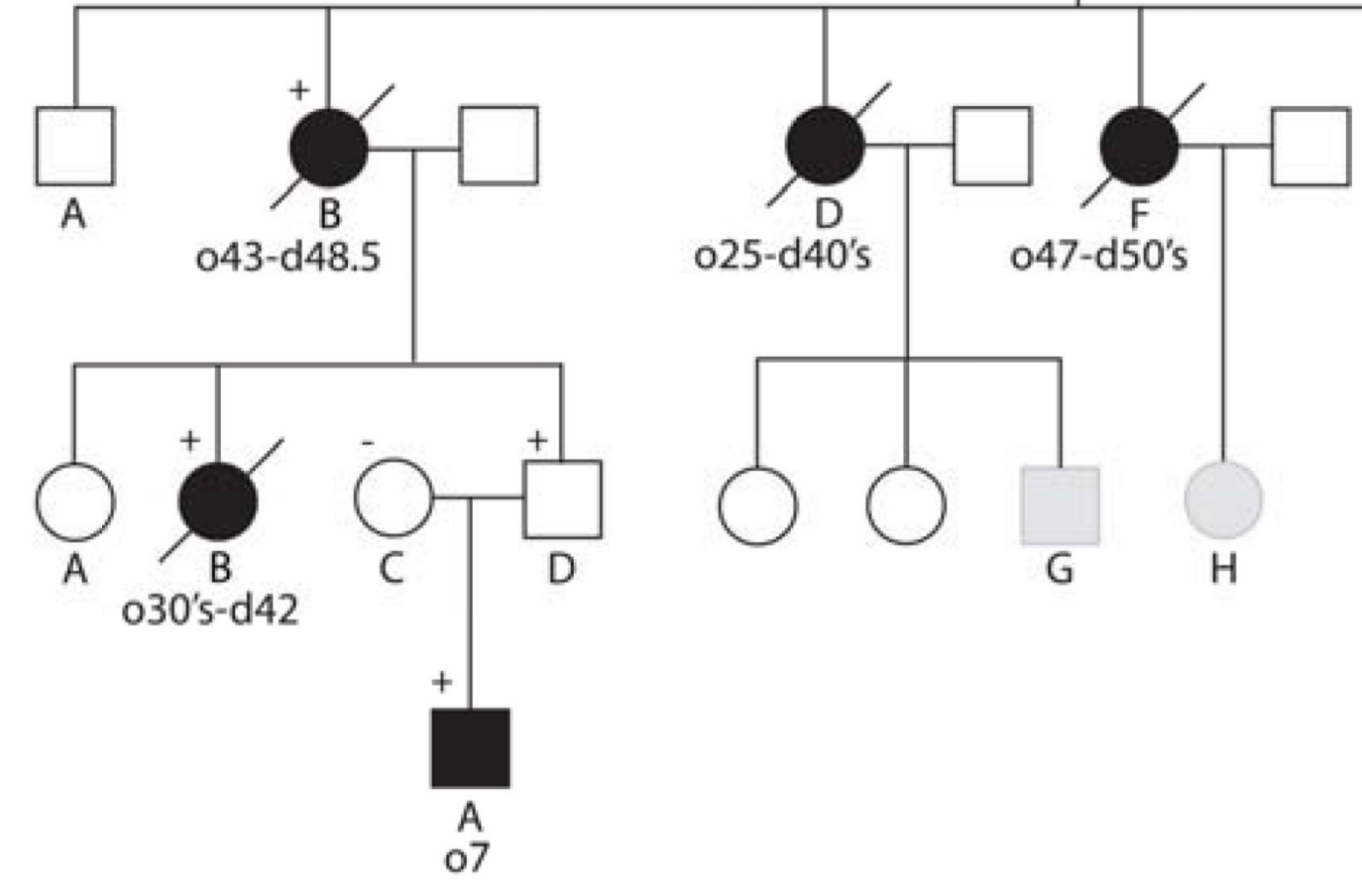

VI

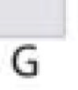

H 
C
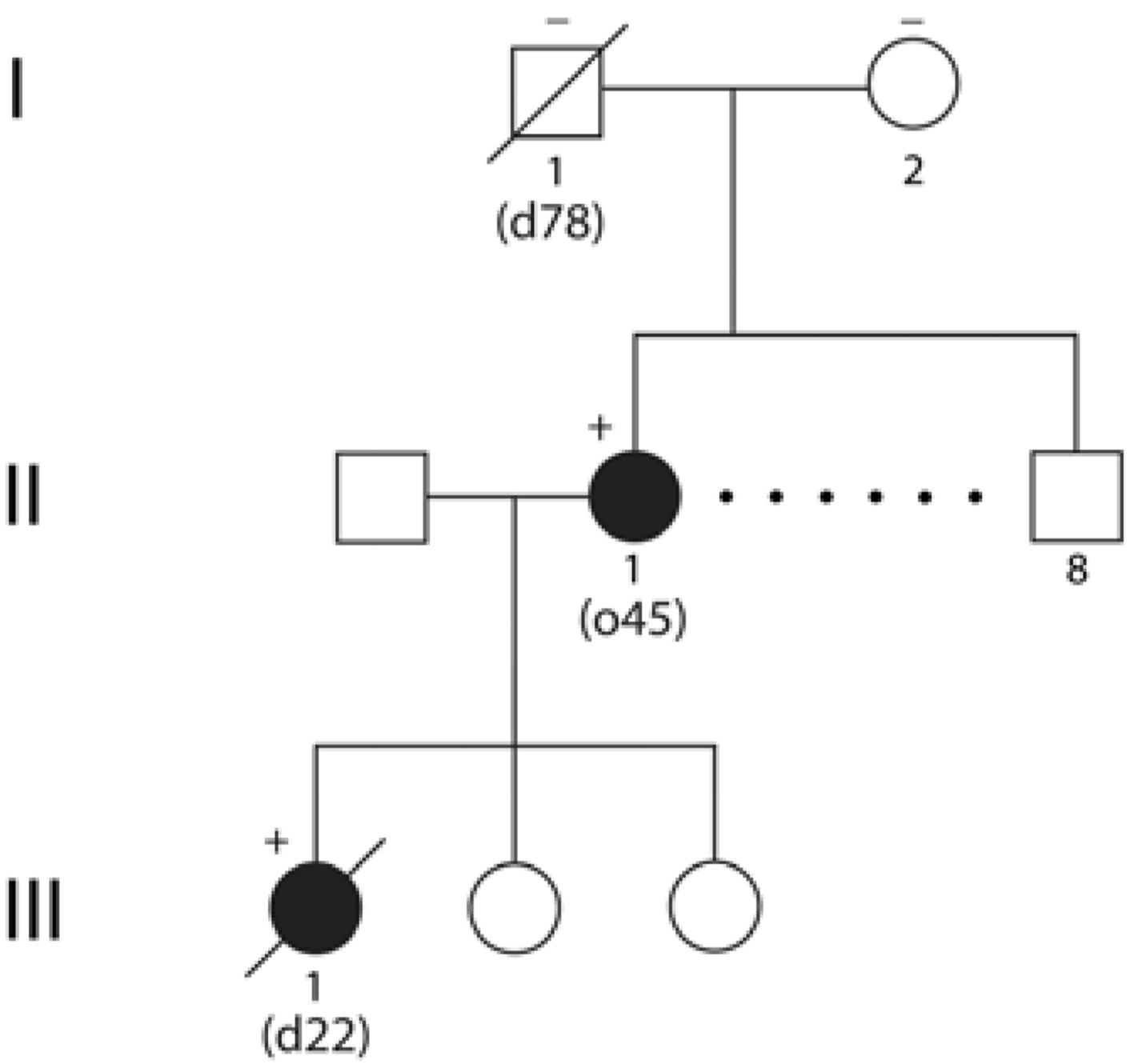

(d78)

Figure 1.

Pedigrees of three families with Alexander disease. Numbers below each symbol indicate the age of onset (o) and the age of most recent report or death (d) (in years). Genotypes, where available, are indicated by a + or - sign above a symbol. Affected individuals are indicated by filled symbols, and individuals with relevant clinical signs and considered probably affected are indicated by gray shading. A) Siblings and parents of the two patients initially reported by Duckett et al. ${ }^{3}$ (II.c and II.d in this diagram). Birth order has been scrambled to protect confidentiality. B) Four generational family related to the adult-onset patients originally reported by Seil et al. ${ }^{2}$ (III.F in this diagram) and Schwankhaus et al. ${ }^{8}$ (IV.B, IV.D, and IV.F in this diagram), continuing the nomenclature used by Schwankhaus 
et al. ${ }^{8}$. Generations I-II of this original pedigree contained no informative individuals, and are not illustrated. C) Two generation family (not previously described). 


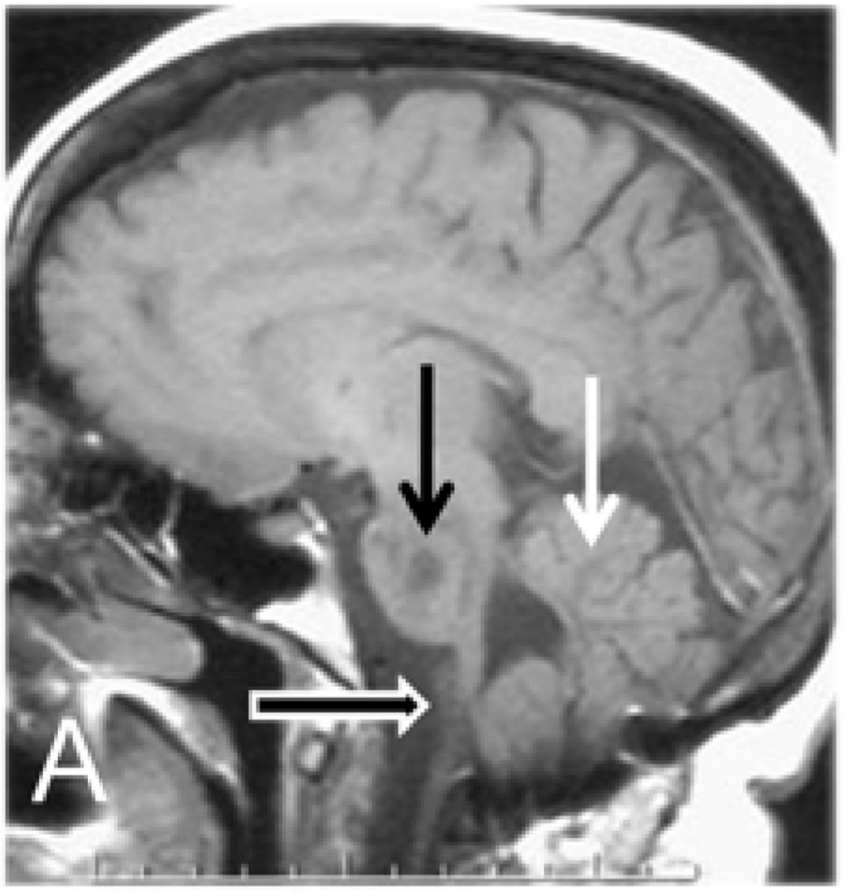

II.C

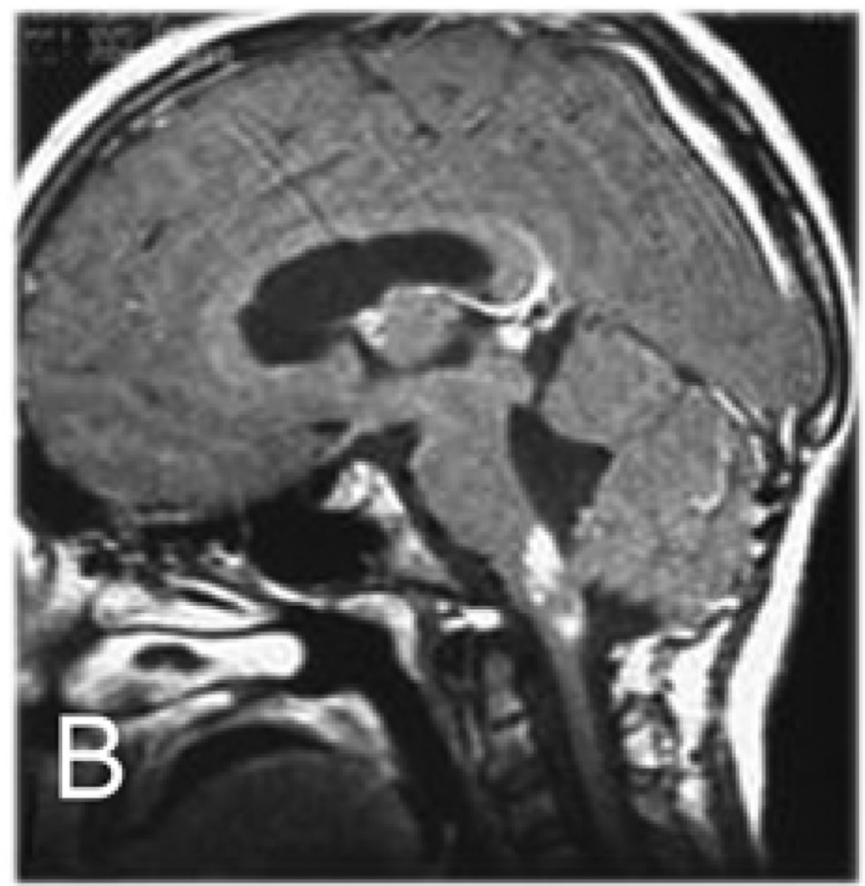

II.d

Figure 2.

MRI images of two affected members of family A. A) Sagittal T1-weighted image of patient II.c, at age 46 yr., showing a lesion in the pons (dark arrow) and severe atrophy of the medulla oblongata (open white arrow). The cerebellar white matter (closed white arrow) has a low signal, which is abnormal. B) Sagittal T1-weighted image after contrast of patient II.d, at age 15 yr., showing an enhancing lesion in the dorsal brain stem. Previous MRIs reported by Duckett et al. ${ }^{3}$ for this patient indicated a space-occupying mass in the left cerebellar hemisphere and signal changes in the deep cerebellar white matter. Patients II.d and II.f had no significant changes in cerebral white matter. 

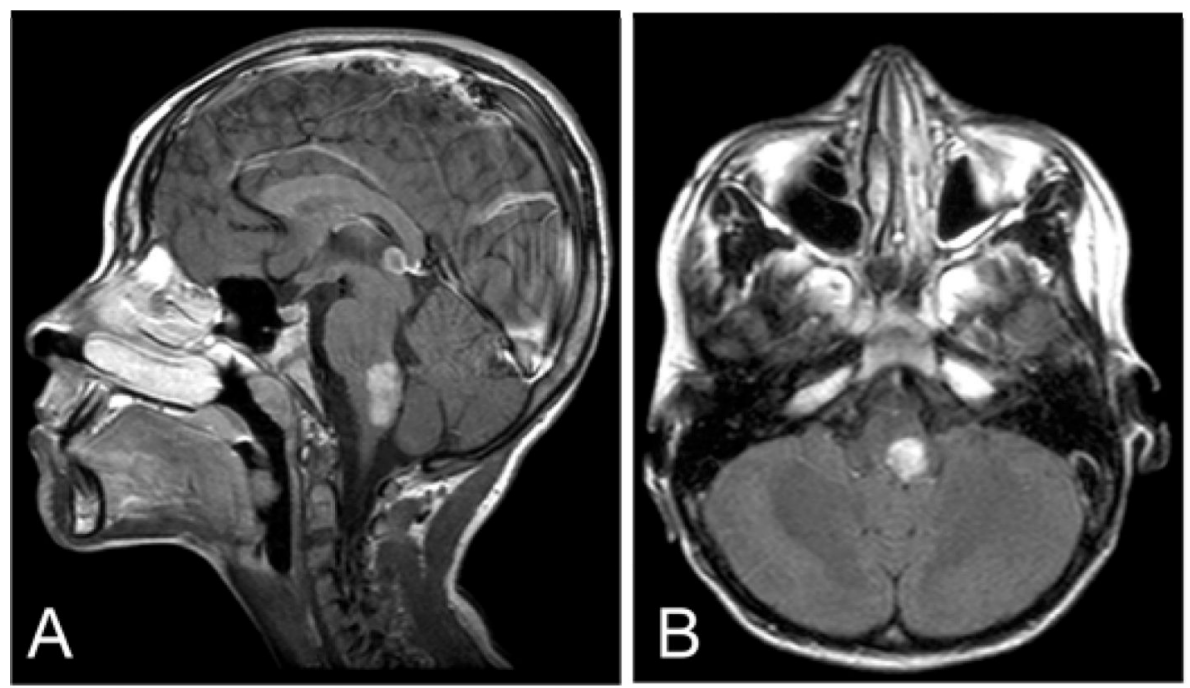

VI.A

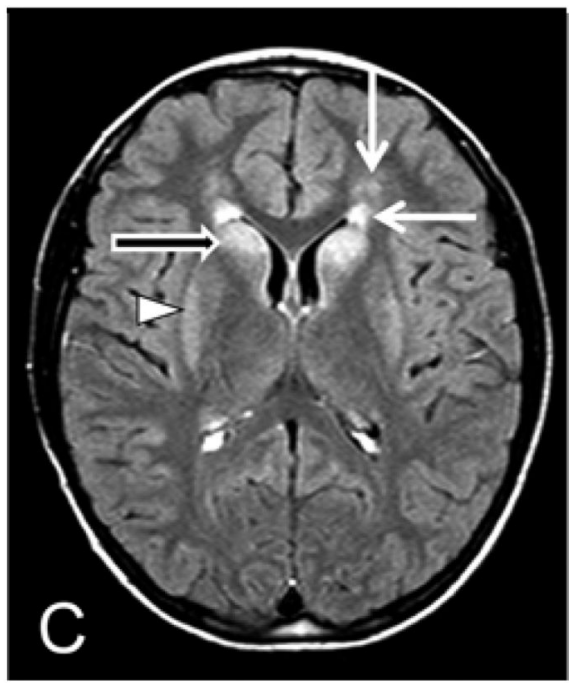

C

Figure 3.

MRI images from patient VI.A of family B at age $8.5 \mathrm{yrs}$. A) Sagittal T1-weighted image after contrast reveals an enhancing tumor-like lesion in the dorsal part of the medulla and lower pons. B) Axial FLAIR image shows the mass lesion in the medulla in the left posterior part. C) Axial FLAIR image shows signal abnormalities in the frontal white matter (closed arrows), head of the caudate nucleus (open arrow) and putamen (arrowhead). 

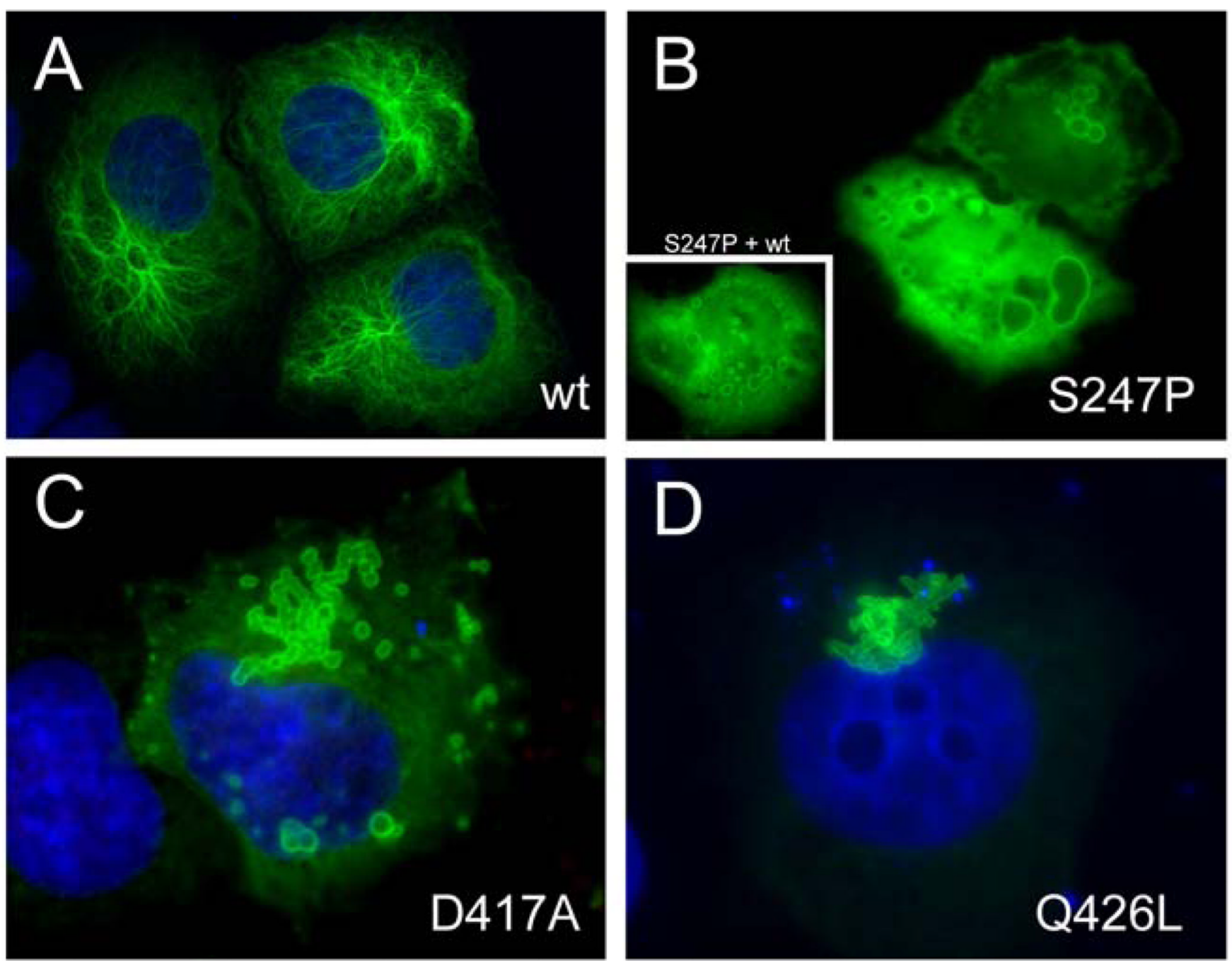

Figure 4.

Effects of pSer247Pro, p.Asp417Ala, and p.Gln426Leu mutations on filament assembly in transfected SW13vim ${ }^{-}$cells. DAPI staining in A, C, and D indicates the position of nuclei. A. Transfection with the wild type GFAP expression vector. A normal filamentous assembly pattern is seen, consisting of a fine network with or without diffuse background in $~ 90 \%$ of cells. B. Transfection with vectors expressing p.Ser247Pro (B), p.Asp417Ala (C), or p.GlnP.GLN426LEUeu (D), each producing ring-like filaments on a background of diffuse staining. The inset in panel B shows results for co-transfection with an equal amount of wild type GFAP expression vector, demonstrating the dominant effect of the mutation. 